\title{
Falls and peripheral nerve injuries: an age-dependent relationship
}

\author{
*Kimon Bekelis, MD,, Symeon Missios, MD, ${ }^{2}$ and Robert J. Spinner, MD² \\ 1Section of Neurosurgery, Dartmouth-Hitchcock Medical Center, Lebanon, New Hampshire; '2Department of Neurosurgery, \\ Louisiana State University-Health Sciences Center, Shreveport, Louisiana; and ${ }^{3}$ Departments of Neurosurgery and Orthopedics, \\ Mayo Clinic, and Department of Anatomy, Mayo Medical School, Rochester, Minnesota
}

\begin{abstract}
OBJECT Despite the growing epidemic of falls, the true incidence of peripheral nerve injuries (PNIs) in this patient population remains largely unknown.

METHODS The authors performed a retrospective cohort study of 839,210 fall-injured patients who were registered in the National Trauma Data Bank (NTDB) between 2009 and 2011 and fulfilled the inclusion criteria. Regression techniques were used to investigate the association of demographic and socioeconomic factors with the rate of PNIs in this patient population. The association of age with the incidence of PNIs was also investigated.
\end{abstract}

RESULTS Overall, 3151 fall-injured patients (mean age 39.1 years, $33.3 \%$ females) sustained a PNI ( $0.4 \%$ of all falls). The respective incidence of PNIs was 2.7 per 1000 patients for ground-level falls, 4.9 per 1000 patients for multilevel falls, and 4.5 per 1000 patients for falls involving force. This demonstrated a rapid increase in the first 2 decades of life, with a maximum rate of $1.1 \%$ of all falls in the 3 rd decade, followed by a slower decline and eventual plateau in the 7th decade. In a multivariable analysis, the association of PNIs with age followed a similar pattern with patients 20-29 years of age, demonstrating the highest association (OR 2.34 [95\% Cl 2.0-2.74] in comparison with the first decade of life). Falls involving force (OR 1.25 [95\% Cl 1.14-1.37] in comparison with multilevel falls) were associated with a higher incidence of PNIs. On the contrary, female sex (OR 0.87 [95\% Cl 0.80-0.84]) and ground-level falls (OR 0.79 [95\% Cl 0.72-0.86]) were associated with a lower rate of PNIs.

CONCLUSIONS Utilizing a comprehensive national database, the authors demonstrated that PNIs are more common than previously described in fall-injured patients and identified their age distribution. These injuries are associated with young adults and falls of high kinetic energy.

http://thejns.org/doi/abs/10.3171/2014.11.JNS142111

KEY WORDS peripheral nerve injury; falls; elderly; NTDB

$\mathrm{T}$ HERE is growing concern over the increasing prevalence of falls. ${ }^{2,4,23,26}$ Especially in the elderly, falls have turned into an epidemic associated with significant morbidity and mortality., ${ }^{4,6}$ Although life-threatening injuries such as subdural hematomas and hip fractures attract a lot of attention, ${ }^{4,26}$ less clinically pronounced injuries, such as peripheral nerve injuries (PNIs), have largely stayed out of the spotlight. PNIs do not contribute to immediate mortality, but they can significantly impact the long-term quality of life of the affected individuals. ${ }^{5}$ PNIs result in major social and economic burden. Functional disability associated with nerve injuries can be devastating. ${ }^{5}$ Their repair frequently requires complicated operations, with variable functional improvement. ${ }^{8}$ Despite their negative impact on long-term population health, the prevalence of PNIs in fall-injured patients and the factors associated with their occurrence in this group are largely unknown. Especially in the setting of the changing demographics of fall-injured patients, ${ }^{4,26}$ the study of the agedependent relationship of PNIs with falls is of particular importance.

PNIs are frequently described in the setting of high-

ABBREVIATIONS BPI = brachial plexus injury; GCS = Glasgow Coma Scale; ISS = Injury Severity Score; NTDB = National Trauma Data Bank; PNI = peripheral nerve injury.

SUBMITTED September 9, 2014. ACCEPTED November 6, 2014.

INCLUDE WHEN CITING Published online May 15, 2015; DOI: 10.3171/2014.11.JNS142111.

DISCLOSURE The authors report no conflict of interest concerning the materials or methods used in this study or the findings specified in this paper.

${ }^{*}$ Drs. Bekelis and Missios contributed equally to this work. 
speed trauma. Brachial plexus injuries (BPIs) in adults have been identified in more than $1 \%$ of multitrauma patients in a single-institution study by Midha. ${ }^{19}$ A similar incidence has been reported for all categories of PNIs in regional experiences, without a focus on fall-injured patients. ${ }^{6,17,20,25}$ In children, PNIs are commonly associated with trauma during birth. Several authors ${ }^{18}$ have shown the prevalence of birth-related PNIs to be around $0.1 \%$. Dorsi et al., 7 in a registry study, identified PNIs in $0.1 \%$ of trauma victims younger than 20 years old. These analyses did not focus on fall-injured patients, were limited by selection bias, and were lacking adjustment for the physiological characteristics of this patient population.

We investigated the age-dependent incidence of PNIs in fall-injured patients and their association with several individual characteristics. We used a regression model in a cohort of patients who sustained falls (ground level, multilevel, and involving external force), and whose data were recorded in the 2009-2011 National Trauma Data Bank (NTDB). The advantage of using a comprehensive database, such as the NTDB, is that it provides an adequate event volume to study a rare entity, such as a PNI.

\section{Methods NTDB}

All individuals sustaining falls who were registered in the NTDB (Committee on Trauma, American College of Surgeons) between 2009 and 2011 were included in the analysis. The NTDB is the largest collection of US trauma registry data ever assembled. It includes data collected from over 900 institutions in the United States. ${ }^{1}$ We merged the available data sets for the variables of interest, using source codes provided by the NTDB. ${ }^{11}$ More information about the NTDB is available at http://www.facs. org/trauma/ntdb/.

\section{Cohort Definition}

To establish the cohort of patients, we used ICD-9 E-codes to identify patients in the registry who were involved in falls. E-codes 833.7, 834.7, 835.7, 880, 880.1, 880.9, 881, 881.1, 882, 883, 883.1, 883.2, 883.9, 884, 884.1, 884.2, 884.3, 884.4, 884.5, 884.6, 884.9, 885, 885.1, 885.2, 885.3, 885.4, 885.9, 886, 886.9, 888, 888.1, 888.8, 888.9, 957, 957.1, 957.2, 957.9, 968.1, 987, 987.1, 987.2 and 987.9 were eligible for inclusion. Exclusion criteria consisted of patients who were dead on arrival to the emergency department, and incomplete record information on mechanism of injury and age.

\section{Primary Outcome and Exposure Variables}

The primary outcome variable was the incidence of PNI. This was defined as an ICD-9 code of 953.x, 954.x, 955.x, 956.x, or 957.x. The effect on the outcome of the pertinent exposure variables was examined. Age was broken down in decades ranging from 0 to 89 years and was treated as a categorical variable. Systolic blood pressure, heart rate, temperature on arrival to the emergency department, and number of neurosurgeons per hospital were continuous variables. Sex, race (Caucasian, African American, Hispanic, Asian, and other), insurance (private insurance, self-pay, Medicaid, Medicare, and other) and fall mechanism (multilevel, ground level, and involving external force) were categorical variables. Glasgow Coma Scale (GCS) score and the patient's Injury Severity Score (ISS) were ordinal variables. The hospital characteristics used in the analysis as categorical variables included hospital region (West, South, Midwest, and Northeast), hospital bedsize ( $<200,200-400,400-600$, and $>600$ beds), and location and teaching status of the hospital (university, urban nonteaching, and community).

Variables were included in the final models after ensuring that the proportion of missing data points was less than $20 \%$. Illicit drug use, alcohol use, respiratory rate, $\mathrm{O}_{2}$ saturation, total elapsed emergency medical services time from dispatch to the emergency department, emergency medical services time at the scene, and patient days in the ICU and/ or on a ventilator were excluded because they were found to have a prevalence of missing data greater than $20 \%$.

\section{Statistical Analysis}

Continuous variables are presented as mean and standard deviation, whereas categorical values are presented as percentages. Continuous variables were compared using the Student t-test, and categorical variables were compared using the chi-square test.

Multiple imputation was performed for each variable associated with missing values using the Amelia II package $^{12}$ in the 64-bit version of R 3.0.2 (R Foundation for Statistical Computing). Imputation was used for the following missing data: sex, payer source, race, ISS, systolic blood pressure, heart rate, temperature, and GCS score. First, the proportion of missing data for variables of interest was calculated. The Amelia II program was used to impute missing data based on the other available variables. This process was repeated 5 times, creating 5 separate imputed data sets. These 5 data sets were combined to create a full-pooled data set with no missing values, which was used in a multinomial logistic regression (logit) model using the Zeilig statistical package ${ }^{13,14}$ in the 64-bit version of $\mathrm{R}$ 3.0.2. The presence or absence of PNIs was used as the dependent variable, and the age group and demographic and socioeconomic factors were used as the independent variables in the regression. The regression was performed on the entire fall cohort.

The initial cohort was then separated, depending on the mechanism of the fall, into multilevel falls, ground-level falls, and falls involving external force groups. Multiple imputation was performed for each cohort, as described above, and a similar multivariable logistic regression was performed for each separate fall mechanism cohort.

As part of the sensitivity analysis, the regressions were repeated without prior multiple imputation, using listwise deletion instead. This resulted in the exclusion of approximately $30 \%$ of the patients. The odds ratios and patterns of variable significance were similar to the results of the imputed cohorts and are therefore not presented below.

The total number of falls per age group was calculated and is presented as a histogram (Fig. 1). The incidence of fall-associated PNIs was also calculated for each age group for the total cohort and for each of the fall mechanism-specific cohorts and depicted in scatter plots, to ex- 


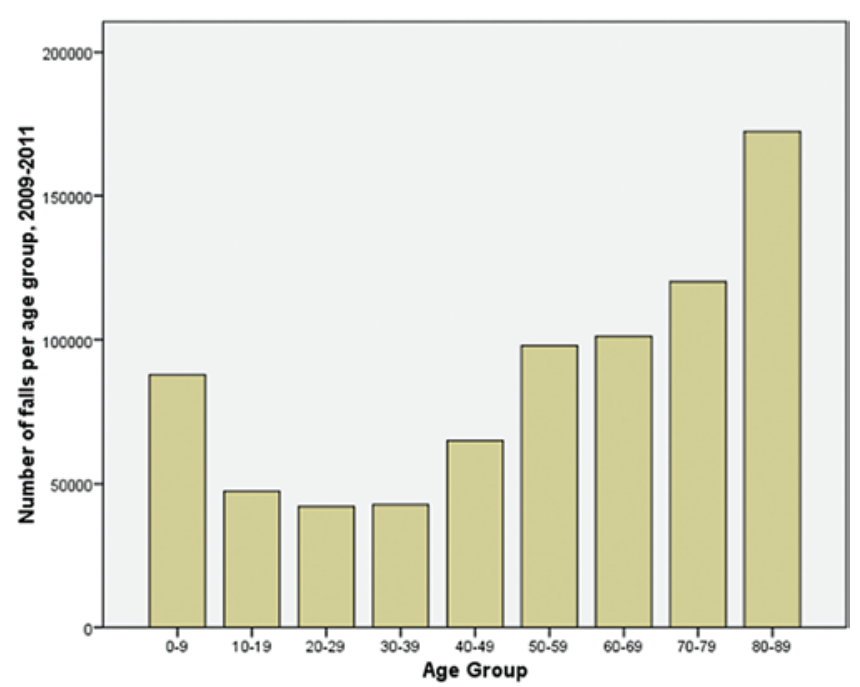

FIG. 1. Histogram demonstrating the incidence of falls per decade of life in our cohort of patients. Figure is available in color online only.

plore the shape of the association. These plots were fitted to a cubic model.

Statistical analyses were performed using the 64-bit version of R 3.0.2 (R Foundation for Statistical Computing) and SPSS version 20 (IBM). All probability values are the results of 2 -sided tests, and the level of significance was set at $\mathrm{p}<0.05$.

\section{Results \\ Patients}

From 2009 to 2011, there were 839,210 individuals involved in falls who were registered in the NTDB and met the inclusion criteria for the study. The age-related distribution of falls is demonstrated in Fig. 1. Of these patients, 316,042 sustained ground-level falls, 338,753 sustained multilevel falls, and 184,415 sustained falls involving an external force (i.e., being pushed). The distribution of exposure variables among patients with and without PNIs can be found in Table 1 .

\section{Primary Outcome}

Overall, 3151 fall-injured patients (mean age 39.1 years, $33.3 \%$ females) sustained a PNI ( $0.4 \%$ of all falls). The respective incidence of PNIs was 2.7 per 1000 patients sustaining ground-level falls, 4.9 per 1000 patients sustaining multilevel falls, and 4.5 per 1000 patients sustaining falls involving force. Table 2 demonstrates the incidence of nerve injuries in the various subgroups, stratified by age.

Overall, 569 patients developed plexus injuries (269 injuries in the brachial plexus, 36 in the lumbosacral plexus, and 264 unspecified), and 1175 developed injuries (sometimes multiple) distal to the plexus (2041 injuries in the upper extremity, 387 in the lower extremity, and 154 unspecified). The respective incidence of BPIs was $0.02 \%$ for ground-level falls, $0.04 \%$ for multilevel falls, and $0.02 \%$ for falls involving external force.

\section{PNI and Age Correlation}

The incidence of PNI demonstrated a rapid increase in the first 2 decades of life, with a maximum rate of $1.1 \%$ of all falls in the 3rd decade (Fig. 2). Subsequently, there was a slower decline in the incidence with a plateau for patients older than 70 years. The correlation of age with incidence of PNI followed a nonlinear polynomial curve, consistent with a cubic model $\left(\mathrm{R}^{2}=0.882\right)$. Similar correlations were observed for multilevel falls $\left(\mathrm{R}^{2}=0.797\right)$ (Fig. 3A), ground-level falls $\left(\mathrm{R}^{2}=0.689\right)$ (Fig. 3B), and falls involving force $\left(\mathrm{R}^{2}=0.780\right)$ (Fig. 3C).

\section{Multivariable Analysis}

The association of PNIs with age and several demographic and socioeconomic factors was examined in a multivariable logistic regression analysis (eTables 1-4 [available online]). Figure 4 demonstrates the distribution of the odds ratios of the association between incidence of PNI and age. The 3rd decade of life demonstrated the highest association (OR 2.34 [95\% CI 2.00-2.74] in comparison with the 1st decade). Increased GCS score on arrival to the emergency department (OR 1.12 [95\% CI 1.09-1.16]), falls involving external force (OR 1.25 [95\% CI 1.14-1.37] in comparison with multilevel falls), university teaching hospitals (OR 1.17 [95\% CI 1.08-1.27]), and increased number of neurosurgeons in the trauma center (OR 1.03 [95\% CI 1.02-1.04]) were associated with a higher incidence of PNIs. On the contrary, female sex (OR 0.87 [95\% CI 0.80-0.84]), ground-level falls (OR 0.79 [95\% CI 0.72-0.86]), and urban nonteaching hospitals (OR 0.85 [95\% CI 0.75-0.96]) were associated with a lower rate of PNIs.

\section{Discussion}

In this study of a comprehensive national database, we demonstrated that PNIs are consistently encountered even in the low-energy trauma associated with falls. They were observed across all fall mechanisms and age groups. A particular age-dependent association was identified. The growing epidemic of falls has been receiving national attention recently. In 2006 alone, 5.8 million Americans older than 65 years sustained a fall. ${ }^{4}$ The rapid aging of the population and rising rate of arthritis are projected to result in a dramatic increase in the number of falls in the elderly. ${ }^{26}$ The study of initially less clinically pronounced injuries, such as PNIs, is crucial to minimize further morbidity and mortality. In addition, it allows the age-specific allocation of health care resources for fall-injured patients. PNIs are associated with considerable economic burden for society, since they frequently result in multiple restorative operations, extensive rehabilitation, and long-term disability. ${ }^{5,8,16,19-21}$ Depending on the type of injury, several operative approaches are undertaken that range from simple decompression to nerve grafting.

Traumatic PNIs have been studied more extensively in high-speed trauma. In a recent national study ${ }^{3}$ of the NTDB, the incidence of PNIs among adult motor vehicle collision victims ranged between $0.73 \%$ and $0.98 \%$. They have been reported ${ }^{21}$ to occur in up to $5 \%$ of patients in a Level I trauma center, most of them in the setting of multitrauma. About half of these patients are the drivers or passengers of motor vehicles. ${ }^{9,16}$ Investigators attribute 
TABLE 1. Patient and hospital characteristics

\begin{tabular}{|c|c|c|c|c|c|c|c|}
\hline \multirow{3}{*}{ Sample size } & \multicolumn{2}{|c|}{ All Patients } & \multicolumn{2}{|c|}{ Patients w/o PNIs } & \multicolumn{2}{|c|}{ Patients w/ PNIs } & \multirow[t]{2}{*}{$p$ Value } \\
\hline & \multicolumn{2}{|c|}{839,210} & \multicolumn{2}{|c|}{836,059} & \multicolumn{2}{|c|}{3151} & \\
\hline & Mean & SD & Mean & SD & Mean & SD & \\
\hline \multirow[t]{2}{*}{ Age in yrs } & 55.68 & 28.04 & 55.75 & 28.04 & 39.14 & 22.64 & $<0.0001$ \\
\hline & No. of Pts & $\%$ & No. of Pts & $\%$ & No. of Pts & $\%$ & \\
\hline \multicolumn{8}{|l|}{ Sex } \\
\hline $\mathrm{F}$ & 417,439 & 49.77 & 416,390 & 49.83 & 1049 & 33.30 & $<0.0001$ \\
\hline$M$ & 421,311 & 50.23 & 419,210 & 50.17 & 2101 & 66.70 & \\
\hline Unreported data & 460 & & 459 & & 1 & & \\
\hline \multicolumn{8}{|l|}{ Region } \\
\hline Northeast & 166,497 & 19.89 & 165,905 & 19.90 & 592 & 18.87 & 0.138 \\
\hline Midwest & 265,224 & 31.69 & 264,357 & 31.71 & 867 & 27.64 & $<0.0001$ \\
\hline South & 253,670 & 30.31 & 252,735 & 30.31 & 935 & 29.81 & 0.497 \\
\hline West & 151,530 & 18.11 & 150,787 & 18.08 & 743 & 23.69 & $<0.0001$ \\
\hline Unreported data & 2289 & & 2275 & & 14 & & \\
\hline \multicolumn{8}{|l|}{ Payer } \\
\hline Medicare & 337,933 & 44.75 & 337,500 & 44.86 & 433 & 15.37 & $<0.0001$ \\
\hline Medicaid & 86,244 & 11.42 & 85,807 & 11.40 & 437 & 15.51 & $<0.0001$ \\
\hline Private payer & 206,899 & 27.40 & 205,836 & 27.36 & 1063 & 37.74 & $<0.0001$ \\
\hline Self-payer & 64,951 & 8.60 & 64,497 & 8.57 & 454 & 16.12 & $<0.0001$ \\
\hline Other & 59,154 & 7.83 & 58,724 & 7.81 & 430 & 15.26 & $<0.0001$ \\
\hline Unreported data & 84,029 & & 83,695 & & 334 & & \\
\hline \multicolumn{8}{|l|}{ Race } \\
\hline Caucasian & 641,477 & 79.40 & 639,280 & 79.42 & 2197 & 73.09 & $<0.0001$ \\
\hline African American & 65,286 & 8.08 & 64,982 & 8.07 & 304 & 10.11 & $<0.0001$ \\
\hline Hispanic & 63,784 & 7.89 & 63,445 & 7.88 & 339 & 11.28 & $<0.0001$ \\
\hline Asian & 13,634 & 1.69 & 13,595 & 1.69 & 39 & 1.30 & 0.085 \\
\hline Other & 23,769 & 2.94 & 23,642 & 2.94 & 127 & 4.22 & $<0.0001$ \\
\hline Unreported data & 31,260 & & 31,115 & & 145 & & \\
\hline \multicolumn{8}{|l|}{ Hospital status } \\
\hline Community & 338,522 & 40.34 & 337,420 & 40.36 & 1102 & 34.97 & $<0.0001$ \\
\hline Nonteaching & 141863 & 16.90 & 141,506 & 16.93 & 357 & 11.33 & $<0.0001$ \\
\hline University & 358,825 & 42.76 & 357,133 & 42.72 & 1692 & 53.70 & $<0.0001$ \\
\hline \multicolumn{8}{|l|}{ Bedsize } \\
\hline$<200$ & 73,767 & 8.79 & 73,544 & 8.80 & 223 & 7.08 & 0.001 \\
\hline $200-400$ & 260,790 & 31.08 & 259,938 & 31.09 & 852 & 27.04 & $<0.0001$ \\
\hline $400-600$ & 235,824 & 28.10 & 234,886 & 28.09 & 938 & 29.77 & 0.037 \\
\hline \multirow[t]{2}{*}{$>600$} & 268,829 & 32.03 & 267,691 & 32.02 & 1138 & 36.12 & $<0.0001$ \\
\hline & Mean & SD & Mean & $\mathrm{SD}$ & Mean & $\mathrm{SD}$ & \\
\hline No. of neurosurgeons & 5.02 & 3.24 & 5.01 & 3.24 & 5.42 & 3.22 & $<0.0001$ \\
\hline No. of trauma surgeons & 6.44 & 2.91 & 6.44 & 2.91 & 6.95 & 3.15 & $<0.0001$ \\
\hline $\mathrm{SBP}$ in $\mathrm{mm} \mathrm{Hg}$ & 140.22 & 28.89 & 140.25 & 28.90 & 134.09 & 24.81 & $<0.0001$ \\
\hline Unreported data, no. of pts & \multicolumn{2}{|c|}{38,407} & \multicolumn{2}{|c|}{38,292} & \multicolumn{2}{|c|}{115} & \\
\hline GCS score & 14.37 & 2.19 & 14.37 & 2.19 & 14.68 & 1.67 & $<0.0001$ \\
\hline Unreported data, no. of pts & \multicolumn{2}{|c|}{86,570} & \multicolumn{2}{|c|}{86,290} & & & \\
\hline HR beats/min & 87.55 & 21.94 & 87.54 & 21.95 & 89.02 & 20.23 & $<0.0001$ \\
\hline Unreported data, no. of pts & & & & & & & \\
\hline Temperature in ${ }^{\circ} \mathrm{C}$ & 36.41 & 1.71 & 36.41 & 1.71 & 36.44 & 1.71 & 0.359 \\
\hline Unreported data, no. of pts & & & & & & & \\
\hline
\end{tabular}


TABLE 1. Patient and hospital characteristics (continued)

\begin{tabular}{|c|c|c|c|c|c|c|c|}
\hline \multirow[t]{2}{*}{ Variable } & \multicolumn{2}{|c|}{ All Patients } & \multicolumn{2}{|c|}{ Patients w/o PNIs } & \multicolumn{2}{|c|}{ Patients w/ PNIs } & \multirow[t]{2}{*}{ p Value } \\
\hline & No. of Pts & $\%$ & No. of Pts & $\%$ & No. of Pts & $\%$ & \\
\hline Alcohol use & 66,909 & 7.97 & 66,571 & 9.66 & 338 & 12.86 & $<0.0001$ \\
\hline Unreported data & 147,591 & & 147,069 & & 522 & & \\
\hline \multicolumn{8}{|l|}{ ISS } \\
\hline $1-24$ & 798,173 & 95.11 & 795,145 & 95.11 & 3028 & 96.10 & 0.010 \\
\hline $24-49$ & 39,916 & 4.76 & 39,801 & 4.76 & 115 & 3.65 & 0.003 \\
\hline $50-75$ & 1121 & 0.13 & 1113 & 0.13 & 8 & 0.25 & 0.064 \\
\hline \multicolumn{8}{|l|}{ Type of fall } \\
\hline Multilevel & 338,753 & 40.37 & 337,142 & 40.33 & 1611 & 51.13 & $<0.0001$ \\
\hline Ground level & 316,042 & 37.66 & 315,253 & 37.71 & 789 & 25.04 & $<0.0001$ \\
\hline Falls involving external force & 184,415 & 21.97 & 183,664 & 21.97 & 751 & 23.83 & 0.012 \\
\hline
\end{tabular}

$\mathrm{HR}=$ heart rate; $\mathrm{pt}=$ patient; $\mathrm{SBP}=$ systolic blood pressure.

only a small percentage of PNIs to falls, not exceeding $15 \%$ in some series. ${ }^{6,17,20,25}$ Most of these studies involve retrospective analyses of single-institution or regional experiences, ${ }^{6,17,20,25}$ with limited generalization, given their inherent selection bias. Our national data demonstrate that this number likely underestimates the true incidence of PNIs in fall victims. The overall incidence of PNIs $(0.4 \%$ for all falls, approaching $1 \%$ in some age groups) in our cohort of fall patients is lower but still comparable to the incidence in motor vehicle collision victims, reported previously in the NTDB. ${ }^{3}$ This observation should increase the alertness of providers for early identification and appropriate treatment of these injuries, to prevent long-term disability.

The age-dependent incidence of traumatic PNIs in fall victims has not been described before. We demonstrated that older age was associated with a rapid increase in the incidence of PNIs, which peaks around the 3rd decade of life. This was followed by a slower decline and eventual stabilization for patients older than 70 years. This might be partially explained by the fact that young adults are more likely to be involved in high-speed falls and to exhibit riskier behaviors while using alcohol (heavy alcohol consumption or binge drinking) and drugs. ${ }^{15,24}$ However, we observed the same age-dependent distribution of PNI in- cidence for falls of varied kinetic energy, including ground falls, multilevel falls, and falls involving force. Since most PNIs in our study involved upper-extremity nerve injuries, another contributor to the observed age association might lie in the pattern of falls in separate age groups. Young children and the elderly have undeveloped and diminished reflexes, respectively, resulting in less use of their upper extremities for protection during a fall, which can subsequently minimize PNIs. This is particularly important in the elderly, since decreased bone density and muscle mass expose their nerves to higher risk of trauma when their distal extremities are injured.

For the elderly in particular, PNIs have been a largely underreported injury. Although proportionally rare, PNIs can become a significant source of morbidity in this age group, given that $16 \%$ of the US population older than 65 years sustains a fall every 3 months. ${ }^{4}$ Even in our cohort, elderly patients comprised the largest group sustaining falls. PNIs are at risk of being masked by other, more clinically pronounced presentations and can go initially unnoticed in this generally less mobile group of patients. This can be detrimental, given the impact of PNIs on quality of life or their potential contribution to further disability and fatal falls.

High-energy falls involving external force were the

TABLE 2. Age-specific incidence of PNIs related to falls stratified by fall mechanism

\begin{tabular}{cccccc}
\hline & \multicolumn{5}{c}{ Incidence of Fall-Related PNIs (per 1000 pt falls) } \\
\cline { 2 - 5 } Age Group (yrs) & Total & Multilevel Falls & Ground-Level Falls & Falls Involving External Force & p Value* \\
\hline $0-9$ & 4.09 & 3.87 & 3.88 & 5.72 & 0.023 \\
\hline $10-19$ & 8.11 & 8.30 & 5.37 & 11.73 & $<0.0001$ \\
\hline $20-29$ & 11.22 & 9.77 & 8.82 & 19.17 & $<0.0001$ \\
\hline $30-39$ & 9.51 & 9.66 & 6.24 & 14.13 & $<0.0001$ \\
\hline $40-49$ & 6.43 & 7.42 & 4.09 & 7.04 & $<0.0001$ \\
\hline $50-59$ & 4.64 & 5.46 & 3.78 & 4.18 & 0.002 \\
\hline $60-69$ & 2.95 & 3.41 & 2.74 & 2.48 & 0.080 \\
\hline $70-79$ & 1.61 & 1.92 & 1.57 & 0.75 & 0.159 \\
\hline $80-89$ & 0.75 & 0.82 & 0.72 & 4.47 & 0.664 \\
\hline Total & 4.01 & 4.92 & 2.71 & & $<0.0001$ \\
\hline
\end{tabular}

* $p$ values attributed to statistical comparisons using a 3-way cross-tab and chi-square analysis. 


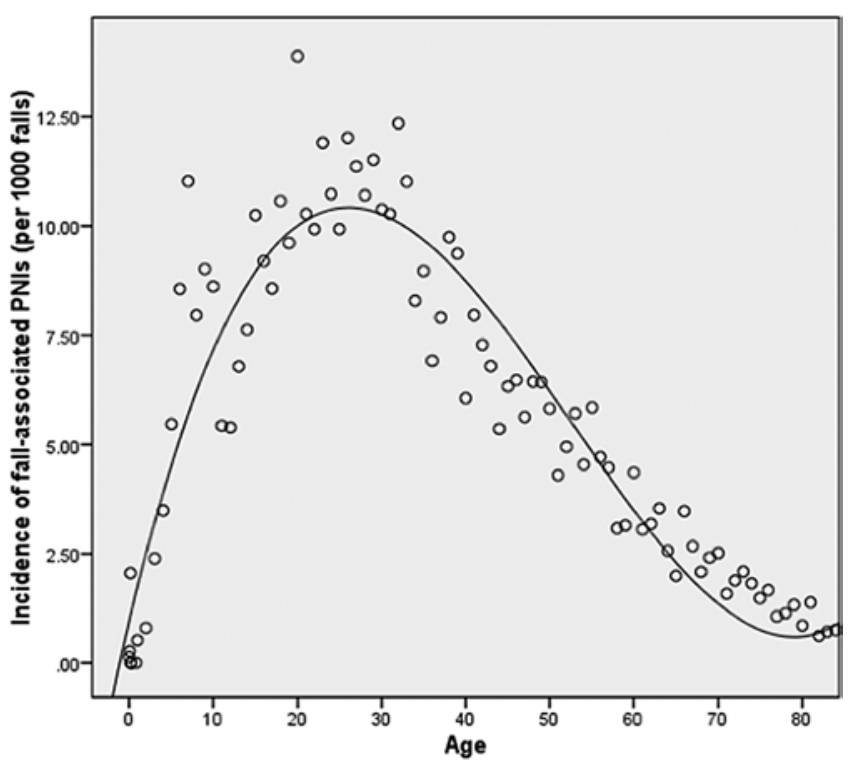

FIG. 2. Scatterplot demonstrating the correlation of patient age with incidence of PNIs for all fall patients in our cohort. This was fitted to a nonlinear polynomial curve, consistent with a cubic model $\left(R^{2}=0.882\right)$.

most common etiology of PNIs in all age groups. This pattern is consistent with prior literature associating PNIs with high-speed collisions. ${ }^{7,19,21}$ Multilevel falls followed in incidence, with ground-level falls being the most rare. The understanding that PNIs can appear with any type of fall will make clinicians more vigilant to diagnose these often occult injuries and protect their patients from secondary complications. In accordance with prior literature ${ }^{7}$ demonstrating that BPIs are more common in high-speed trauma, BPIs represented a minority of injuries in our cohort of fall patients. On the contrary, upper-extremity nerve injuries were very prevalent, potentially caused by patients using their arms as protection during the fall.

Lastly, several other secondary factors demonstrated an association with the rate of PNIs. The association of male sex with PNIs is likely due to riskier behaviors by this subgroup, resulting in more severe trauma. On the other hand, the higher GCS score in patients with PNIs is the result of

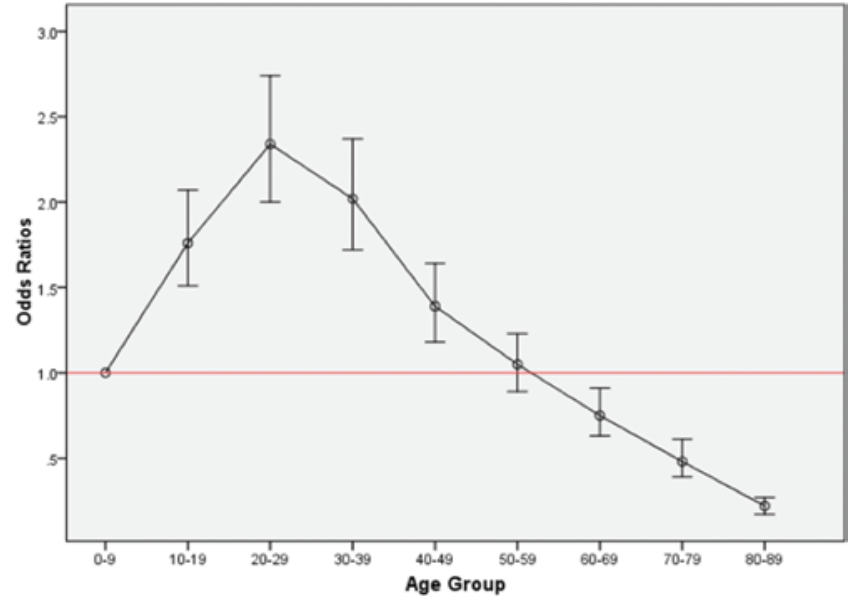

FIG. 4. Association of age with the risk of PNIs for fall-injured patients. Whiskers indicate $95 \% \mathrm{Cl}$. Figure is available in color online only.

survivorship bias. Patients with better neurological examination findings on arrival to the emergency department are more likely to survive and therefore be diagnosed with a PNI. The higher prevalence of PNIs in teaching hospitals with a large number of neurosurgeons is a reflection of a referral bias of the more severe accidents to specialized tertiary care trauma centers and increased diagnostic sensitivity in these institutions. This does not represent a causal relationship.

The present study has several limitations common to administrative databases. First, residual confounding could account for some of the observed associations. Second, coding inaccuracies will undoubtedly occur and can affect our estimates. This is no different from other studies involving the NTDB. Third, the NTDB during the years studied did not include all trauma centers from all states. The NTDB is expected to preferentially involve larger hospitals, as well as younger and more severely injured patients. ${ }^{1}$ Although this introduces a selection bias, the NTDB is the most extensive trauma registry available. Fourth, there is a large number of missing data for some variables in the database. The values missing are not expected to be random. ${ }^{22}$ We addressed this issue by excluding variables with more than $20 \%$ missing
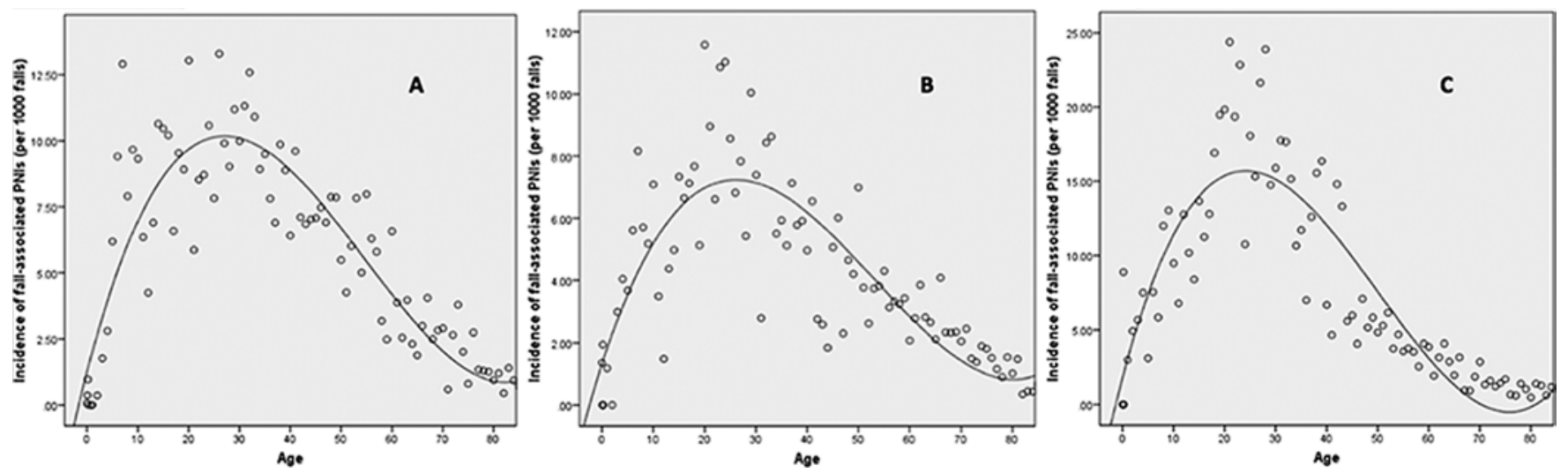

FIG. 3. Scatterplots demonstrating the correlation of patient age with incidence of PNIs for patients in our cohort sustaining multilevel falls (A), ground-level falls (B), and falls involving external force (C). These were fitted to a nonlinear polynomial curve, consistent with a cubic model ( $R^{2}=0.797$ for multilevel falls, $R^{2}=0.689$ for ground-level falls, and $R^{2}=0.780$ for falls involving external force). 
values and used multiple imputation techniques to estimate the rest of the missing values, as previously established for NTDB.$^{10}$ However, this is still inferior to utilizing real data. Fifth, the NTDB is lacking information on the longterm functional status of the patients. Future studies should identify the long-term impact of PNIs on the quality of life of individuals who have fallen. Sixth, the lack of adequate numbers in each subcategory of PNIs did not allow further subgroup analysis. Additionally, NTDB does not specify whether falls involving external force were ground level or multilevel, and therefore further analyses on that matter are limited. Seventh, we used largely ecological data, and therefore causality cannot be established solely based on them.

\section{Conclusions}

Despite the negative impact of PNIs on long-term population health and the rising prevalence of falls, the incidence of PNIs in this group of patients is largely unknown. Utilizing a comprehensive national database, we demonstrated that PNIs are more common than previously identified in fall-injured patients. We identified their relative incidence across several fall mechanisms and age strata. The alertness of providers for early identification and appropriate treatment of these injuries in fall-injured patients should be increased to prevent long-term disability.

\section{References}

1. American College of Surgeons: National Trauma Data Bank: NTDB Research Data Set Admission Year 2011, Annual Report. Chicago: American College of Surgeons, 2011

2. Barbour KE, Stevens JA, Helmick CG, Luo YH, Murphy LB, Hootman JM, et al: Falls and fall injuries among adults with arthritis-United States, 2012. MMWR Morb Mortal Wkly Rep 63:379-383, 2014

3. Bekelis K, Missios S, Spinner R: Restraints and peripheral nerve injuries in adult victims of motor vehicle crashes. J Neurotrauma 31:1077-1082, 2013

4. Centers for Disease Control and Prevention (CDC): Selfreported falls and fall-related injuries among persons aged $>$ or $=65$ years - United States, 2006. MMWR Morb Mortal Wkly Rep 57:225-229, 2008

5. Ciaramitaro P, Mondelli M, Logullo F, Grimaldi S, Battiston B, Sard A, et al: Traumatic peripheral nerve injuries: epidemiological findings, neuropathic pain and quality of life in 158 patients. J Peripher Nerv Syst 15:120-127, 2010

6. Coert JH, Dellon AL: Peripheral nerve entrapment caused by motor vehicle crashes. J Trauma 37:191-194, 1994

7. Dorsi MJ, Hsu W, Belzberg AJ: Epidemiology of brachial plexus injury in the pediatric multitrauma population in the United States. J Neurosurg Pediatr 5:573-577, 2010

8. Dubuisson AS, Kline DG: Brachial plexus injury: a survey of 100 consecutive cases from a single service. Neurosurgery 51:673-683, 2002

9. Eser F, Aktekin LA, Bodur H, Atan C: Etiological factors of traumatic peripheral nerve injuries. Neurol India 57:434437, 2009

10. Galvagno SM Jr, Haut ER, Zafar SN, Millin MG, Efron DT, Koenig GJ Jr, et al: Association between helicopter vs ground emergency medical services and survival for adults with major trauma. JAMA 307:1602-1610, 2012

11. Haider AH, Saleem T, Leow JJ, Villegas CV, Kisat M, Schneider EB, et al: Influence of the National Trauma Data Bank on the study of trauma outcomes: is it time to set re- search best practices to further enhance its impact? J Am Coll Surg 214:756-768, 2012

12. Honaker J, King G, Blackwell M: AMELIA II: A Program for Missing Data. (http://gking.harvard.edu/amelia) [Accessed April 1, 2015]

13. Imai K, King G, Lau O: Zelig: Everyone's Statistical Software. (http://zeligproject.org) [Accessed April 1, 2015]

14. Imai K, King G, Lau O: Toward a common framework for statistical analysis and development. J Comput Graph Stat 17:1-22, 2008

15. Klauer SG, Guo F, Simons-Morton BG, Ouimet MC, Lee SE, Dingus TA: Distracted driving and risk of road crashes among novice and experienced drivers. N Engl J Med 370:54-59, 2014

16. Kouyoumdjian JA: Peripheral nerve injuries: a retrospective survey of 456 cases. Muscle Nerve 34:785-788, 2006

17. Lad SP, Nathan JK, Schubert RD, Boakye M: Trends in median, ulnar, radial, and brachioplexus nerve injuries in the United States. Neurosurgery 66:953-960, 2010

18. Malessy MJ, Pondaag W: Obstetric brachial plexus injuries. Neurosurg Clin N Am 20:1-14, v, 2009

19. Midha R: Epidemiology of brachial plexus injuries in a multitrauma population. Neurosurgery 40:1182-1189, 1997

20. Noble J, Munro CA, Prasad VS, Midha R: Analysis of upper and lower extremity peripheral nerve injuries in a population of patients with multiple injuries. J Trauma 45:116-122, 1998

21. Robinson LR: Traumatic injury to peripheral nerves. Muscle Nerve 23:863-873, 2000

22. Roudsari B, Field C, Caetano R: Clustered and missing data in the US National Trauma Data Bank: implications for analysis. Inj Prev 14:96-100, 2008

23. Socias CM, Chaumont Menéndez CK, Collins JW, Simeonov P: Occupational ladder fall injuries - United States, 2011. MMWR Morb Mortal Wkly Rep 63:341-346, 2014

24. Substance Abuse and Mental Health Services Administration: Results from the 2012 National Survey on Drug Use and Health: Summary of National Findings. Rockville, MD: Substance Abuse and Mental Health Services Administration, Center for Behavioral Health Statistics and Quality, 2012

25. Taylor CA, Braza D, Rice JB, Dillingham T: The incidence of peripheral nerve injury in extremity trauma. Am J Phys Med Rehabil 87:381-385, 2008

26. Tinetti ME, Baker DI, King M, Gottschalk M, Murphy TE, Acampora D, et al: Effect of dissemination of evidence in reducing injuries from falls. N Engl J Med 359:252-261, 2008

\section{Author Contributions}

Conception and design: Bekelis, Spinner. Acquisition of data: Bekelis. Analysis and interpretation of data: Bekelis, Missios. Drafting the article: Bekelis. Critically revising the article: Missios, Spinner. Reviewed submitted version of manuscript: Missios, Spinner. Approved the final version of the manuscript on behalf of all authors: Bekelis. Statistical analysis: Missios. Study supervision: Spinner.

\section{Supplemental Information}

Supplemental material is available with the online version of the article.

eTables 1-4. http://thejns.org/doi/suppl/10.3171/2014.11. JNS142111.

\section{Correspondence}

Kimon Bekelis, Section of Neurosurgery, Dartmouth-Hitchcock Medical Center, One Medical Center Dr., Lebanon, NH 03756. email: kbekelis@gmail.com. 